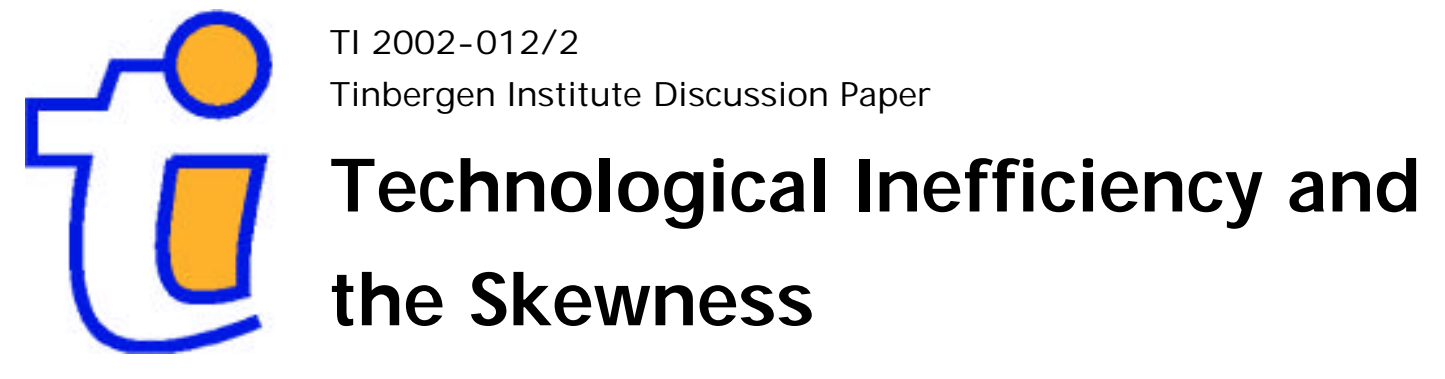

\author{
Martin A. Carree
}

Department of General Economics, Faculty of Economics, Erasmus University Rotterdam, Tinbergen Institute, and

Maastricht University 
Tinbergen Institute

The Tinbergen Institute is the institute for economic research of the Erasmus Universiteit Rotterdam, Universiteit van Amsterdam and

Vrije Universiteit Amsterdam.

Tinbergen I nstitute Amsterdam

Keizersgracht 482

1017 EG Amsterdam

The Netherlands

Tel.: +31.(0)20.5513500

Fax: $\quad+31 .(0) 20.5513555$

Tinbergen Institute Rotterdam

Burg. Oudlaan 50

3062 PA Rotterdam

The Netherlands

Tel.: $\quad+31 .(0) 10.4088900$

Fax: $\quad+31 .(0) 10.4089031$

Most TI discussion papers can be downloaded at

http://www.tinbergen.nl 


\title{
Technological Inefficiency and the Skewness of the Error Component in Stochastic Frontier Analysis
}

\author{
Martin A. Carree \\ Erasmus University Rotterdam \\ and \\ Maastricht University
}

\begin{abstract}
This paper concentrates on negatively skewed one-sided distributions as an explanation of the occurence of positive (negative) skewness in the case of stochastic production (cost) frontier analysis. It takes as example the binomial distribution that can have negative or positive skew and derives the method-of-moments estimators.
\end{abstract}

January 2002

Keywords: Production frontier, cost frontier, skewness, binomial distribution

JEL classification: C13, D24

email: m.carree@mw.unimaas.nl or carree@few.eur.nl

correspondence to:

dr. M.A. Carree

MW-ORG

FdEWB

Maastricht University

PO BOX 616

6200 MD Maastricht

The Netherlands 


\section{Introduction}

A popular econometric technique to estimate the extent of firm inefficiency is stochastic frontier analysis. A pioneering publication on the econometric estimation of stochastic frontiers is Aigner et al. (1977). They suggest an estimation procedure in which a production frontier is estimated along with a two-part composed error term. The first part of the error term consists of conventional statistical noise and is usually assumed to be normally distributed. The second part represents firm inefficiency and is assumed to follow a one-sided distribution. Several distributions have been proposed for the onesided distribution including the half-normal distribution, the truncated normal distribution, the exponential distribution and the two-parameter gamma-distribution (Greene, 1990). Each of these onesided distributions has a positive skewness. $\mathrm{Li}$ (1996) considers the case of the symmetric one-sided uniform distribution.

A common problem in the use of the stochastic production frontier analysis is that the estimated skewness of the residuals is positive. Green and Mayes (1991) report that for a sample of 151 U.K. industries 32\% showed a positive skewness of the combined residual and that for a sample of 140 Australian industries a similar problem was encountered in $35 \%$ of the cases. ${ }^{1}$ A positive skewness is considered problematic because it cannot be reconciled with a one-sided distribution of inefficiencies that is positively skewed. Green and Mayes argue that, apart from possible misspecification of the production functions, this either indicates 'super efficiency' (all firms in the industry are efficient) or the inappropriateness of the technique of frontier production function analysis to measure inefficiencies. They overlook one important additional possibility: that of negatively skewed one-sided distributions of inefficiencies. In this paper we consider this possibility and show that it has important consequences for the interpretation of the skewness of the error term as a measure of technological inefficiency.

\section{The model of stochastic production frontier analysis}

We consider the following production frontier model for a sample of $N$ firms: ${ }^{2}$

$$
y_{i}=\alpha+x_{i}^{\prime} \beta+\varepsilon_{i} \quad i=1, \ldots, N
$$

with $\varepsilon_{i}=v_{i}-u_{i}$ being the composite error term. The commonly made assumption for the statistical noise term $v_{i}$ is that it is i.i.d. $N\left(0, \sigma^{2}\right)$. The $u_{i} \geq 0$ represents the technological inefficiency of firm $i$. The two parts of the error term are assumed to be independently distributed. The $y_{i}$ and the $x_{i}$ -

\footnotetext{
${ }^{1}$ Another example is Mester (1997) who applies the stochastic cost frontier analysis and finds that out of twelve U.S. bank districts three have negatively skewed residuals. She carefully remarks that her "frontier model with normal-half-normal error term does not fit the data in these Districts." (p.238).

${ }^{2}$ Although we concentrate upon the production frontier case, the arguments are similar for the cost frontier model in which the costs of firm $i$ are determined by the cost frontier and an error term of the form $\varepsilon_{i}=u_{i}+v_{i}$ with the one-sided error term $u_{i} \geq 0$ capturing cost inefficiencies.
} 
vector stand for the output and the inputs used in the production process, respectively. The composite error term $\varepsilon_{i}$ has an expected value equal to $E \varepsilon_{i}=-E u_{i}$ and a third central moment equal to:

$E\left(\varepsilon_{i}-E \varepsilon_{i}\right)^{3}=E\left(v_{i}-u_{i}+E u_{i}\right)^{3}=-E\left(u_{i}-E u_{i}\right)^{3}$

Therefore, a positively skewed distribution of the inefficiencies $u_{i}$ implies that the adjusted error term $\varepsilon_{i}-E \varepsilon_{i}$ has a negative skewness. Now it has been common practice to use a positively skewed one-sided distribution. In fact, in case one wants the one-sided error $u_{i}$ to have an unbounded range, then most well-known distributions are in fact positively skewed. Examples include the gamma distribution (including the exponential distribution), the Poisson distribution, the negative binomial distribution, the truncated normal distribution and the half-normal distribution. But there is at least one well-known distribution defined on $[0, \infty)$ that may show negative skewness: the Weibull distribution. ${ }^{3}$ In case one allows for the one-sided error to have a bounded range, a longer list of wellknown distributions with (possible) negative skewness becomes available. In the current paper we will examine the binomial $b(n, p)$-distribution. There is no particular reason to choose this distribution apart from that it allows for both positive and negative skewness.

A simple method-of-moments (MM) estimator for the binomial one-sided distribution can be derived by using the corrected OLS approach (see e.g. Greene, 1990 and Olson et al., 1980). This approach implies that first the parameters of the production function (1) are estimated using least squares and that second the estimated residuals $e_{i}$ are used to estimate the parameters of the distributions of $u_{i}$ and $v_{i}$. The corrected OLS procedure leads to consistent estimators of the parameters of the production function and of the composed error term distribution. ${ }^{4}$ First define $r_{i}=u_{i}-E u_{i}$ so that $E r_{i}=0$. Because $r_{i}$ and $v_{i}$ are independent, we have that $E r_{i}^{j} v_{i}^{k}=E r_{i}^{j} E v_{i}^{k}$ with $j$ and $k$ positive integers. In addition, because $v_{i}$ is distributed symmetrically we have $E v_{i}^{k}=0$ if $k$ is an odd positive integer. From the error decomposition $\varepsilon_{i}-E \varepsilon_{i}=v_{i}-r_{i}$ we find:

$$
E\left(\varepsilon_{i}-E \varepsilon_{i}\right)^{2}=E v_{i}^{2}+E r_{i}^{2}
$$

\footnotetext{
${ }^{3}$ Johnson et al. (1994, p.633) show that a Weibull distribution has a positive skewness for parameter values up to 3.602 and a negative coefficient of skewness for higher parameter values. Li (1996, p.222) does not recognize this possibility and argues that a one-sided error component with unbounded range always has a positive skewness.

${ }^{4}$ The method-of-moment estimators for the two parameters of the binomial distribution are derived using the second, third and fourth sample moments. The use of higher-order moments makes the estimators vunerable to outliers and may lead to poor small sample properties. Greene (1990)'s MM-estimators for the two-parameter Gamma-distribution suffer from this problem as well. Hosking (1990) proposes to use L-moments instead of the standard measures of skewness and kurtosis to achieve relatively small sensitivity to outliers. However, the derivation of estimators based upon L-moments is beyond the scope of the present paper.
} 
$E\left(\varepsilon_{i}-E \varepsilon_{i}\right)^{3}=-E r_{i}^{3}$

$E\left(\varepsilon_{i}-E \varepsilon_{i}\right)^{4}=E v_{i}^{4}+6 E v_{i}^{2} E r_{i}^{2}+E r_{i}^{4}$

For a normally distributed $v_{i}$ we insert $E v_{i}^{2}=\sigma^{2}$ and $E v_{i}^{4}=3 \sigma^{4}$. By combining equations (3) and (5) we have:

$E\left(\varepsilon_{i}-E \varepsilon_{i}\right)^{4}-3\left(E\left(\varepsilon_{i}-E \varepsilon_{i}\right)^{2}\right)^{2}=E r_{i}^{4}-3\left(E r_{i}^{2}\right)^{2}$

From equations (4) and (6) a MM-estimator for a two-parameter distribution can be derived in analogue to Greene (1990). For the binomial distribution we have that $E r_{i}^{2}=n p(1-p)$, $E r_{i}^{3}=n p(1-p)(1-2 p)$ and $E r_{i}^{4}=3(n p(1-p))^{2}+n p(1-p)\left(1-6 p+6 p^{2}\right){ }^{5}$ From the third central moment it is obvious that the binomial distribution has a positive skewness for $p$ between zero and one half and a negative skewness for $p$ between one half and unity. After replacing the $k$-th central moments of $\varepsilon_{i}$ with the sample analogues $\hat{\mu}_{k}=\sum_{i} e_{i}^{k} / N$ we have the following two equations that determine MM-estimates for $n$ and $p$ :

$\hat{\mu}_{3}=-n p(1-p)(1-2 p) \quad \hat{\mu}_{4}-3 \hat{\mu}_{2}^{2}=n p(1-p)\left(1-6 p+6 p^{2}\right)$

That is, the values of $p$ determine the signs of the sample moments $\hat{\mu}_{3}$ (skewness) and $\hat{\mu}_{4}-3 \hat{\mu}_{2}^{2}$ (kurtosis adjusted for the value for normality). Assume that $\hat{\mu}_{3} \neq 0$ and define $x=\left(\hat{\mu}_{4}-3 \hat{\mu}_{2}^{2}\right) / \hat{\mu}_{3}$. According to equation (7) this should be equal to $\left(6 p^{2}-6 p+1\right) /(2 p-1)$. From this we derive the two possible values of $p$ as a function of $x$ :

$p_{1}=\frac{1}{2}+\frac{1}{6} x+\frac{1}{6} \sqrt{x^{2}+3} \quad p_{2}=\frac{1}{2}+\frac{1}{6} x-\frac{1}{6} \sqrt{x^{2}+3}$

For values of $x$ less than -1 only the $p_{1}$-solution is allowed. For values of $x$ in excess of +1 only the $p_{2}$-solution is allowed. For values of $x$ in between -1 and +1 the signs of $\hat{\mu}_{3}$ and $\hat{\mu}_{4}-3 \hat{\mu}_{2}^{2}$ determine which of the two solutions is appropriate. That is, if skewness is positive $\left(\hat{\mu}_{3}>0\right)$ then the $p_{1}$-solution will be chosen otherwise the $p_{2}$-solution. In Figure 1 the graphs of $p_{1}$ and $p_{2}$ as a function of $x$ are given. 


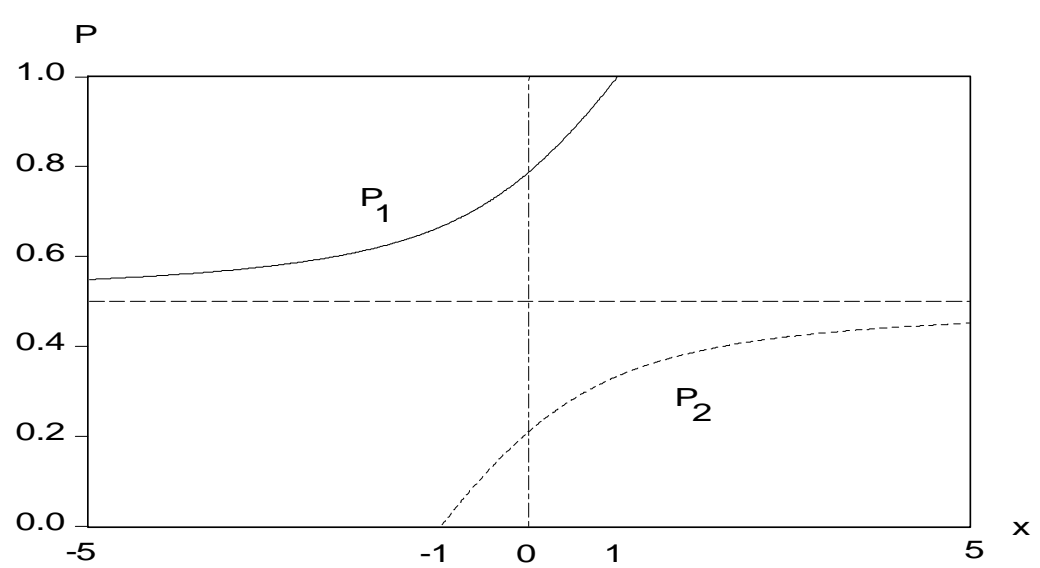

Figure 1: Method-of-moments estimator $p$ as a function of $x=\left(\hat{\mu}_{4}-3 \hat{\mu}_{2}^{2}\right) / \hat{\mu}_{3}$.

Not all combinations of the empirical values for $\hat{\mu}_{3}$ and $\hat{\mu}_{4}-3 \hat{\mu}_{2}^{2}$ allow for MM-estimates. In fact, in case $\hat{\mu}_{4}-3 \hat{\mu}_{2}^{2}>\hat{\mu}_{3}>0$ or $\hat{\mu}_{4}-3 \hat{\mu}_{2}^{2}>-\hat{\mu}_{3}>0$ there are no valid MM-estimates for $p$. To derive the MM-estimator for $E v_{i}^{2}=\sigma^{2}$ using equation (3) we also require that $\hat{\mu}_{2}$ should not be less than $n p(1-p)$ after inserting the MM-estimates of $p$ and $n$. It is a question of empirics whether these violations, which would indicate the implausibility of the one-sided distribution to be of a binomial type, are encountered.

\section{What do negative and positive skewnesses actually measure?}

Empirical studies using the production frontier approach have been assuming positively skewed onesided distributions (and, hence, negatively skewed adjusted composite error terms). As a consequence, when a positive value of $\hat{\mu}_{3}$ was found, the only logical conclusion could be that there had been unfortunate sampling from a distribution that had in fact a population skewness below zero. As Monte Carlo studies have shown, this is a possibility that may occur relatively frequently in case the one-sided distribution has a small variance in comparison with the symmetric error distribution (see e.g. Fan et al., 1996 and Green and Mayes, 1991). Waldman (1982) showed that resorting to a maximum likelihood procedure instead of a corrected OLS procedure does not resolve the problem. In fact, he has shown that, in case of a positive $\hat{\mu}_{3}$, the ML estimator for the stochastic frontier model is simply OLS for the slope vector and the absence of any efficiencies (variance of $u_{i}$ is zero). When an industry showed positive skewness of the residuals it was therefore assumed that there were little if

\footnotetext{
${ }^{5}$ See for example Johnson et al. (1992, p.107).
} 
any inefficiencies. Green and Mayes (1991) argue that a "positive skew implies that establishments in the industry are 'super efficient', rather than inefficient" (p.528).

In contrast to the conclusion of 'super efficiency' in case of a positive skewness, the example of the binomial distribution shows that a positive skewness suggests a one-sided distribution that has low probabilities for small inefficiencies and high probabilities of large inefficiencies. For the binomial distribution it indicates that $p$ is between one half and one. Hence, only a small fraction of the firms or plants attain a level of productivity close to the frontier while a large fraction attains considerable inefficiencies. See Figure 3 in which we have $n$ equal to twenty (inefficiency categories) and $p$ equal to 0.75 . The case of a negative skewness implies that only a small fraction of firms are lagging behind. See Figure 2 in which we have $p$ equal to 0.25 .

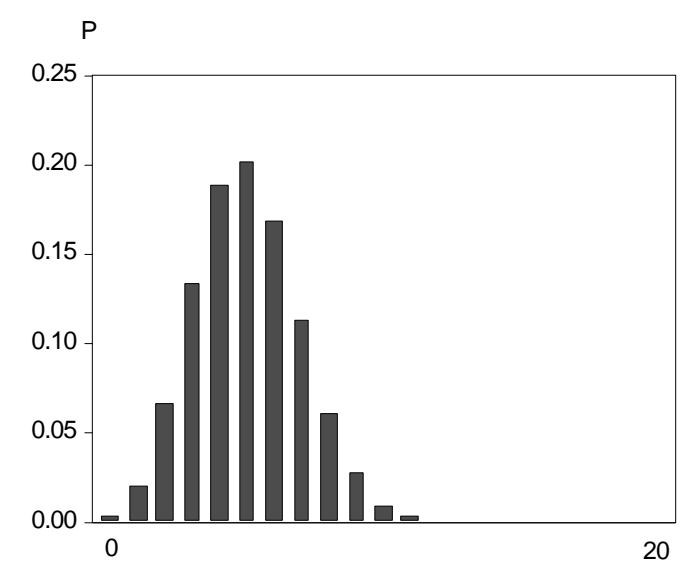

Figure 2: Probabilities of $b(20,0.25)$

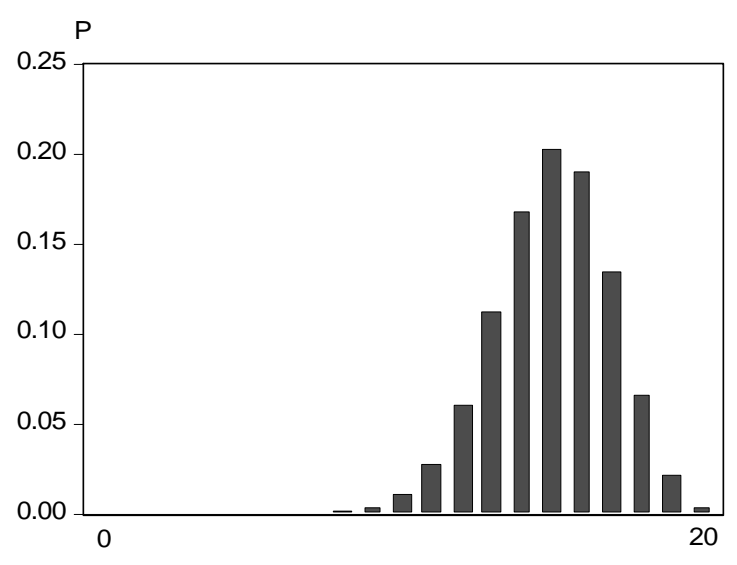

Figure 3: Probabilties of $b(20,0.75)$

Figures 2 and 3 can also be interpreted as two stages in an industry characterized by the cycle of innovation and imitation. Assume that the productivities in an industry are characterized by Figure 2. In case one firm achieves an important innovation by which it can increase productivity, it becomes dominant (in terms of productivity), and Figure 3 may emerge. Other firms will then seek to imitate the successful firm and Figure 2 may be restored. This process of 'transient dominance' in an industry would lead to a cyclical time series pattern of positive and negative skewness of residuals of the stochastic production frontier analysis: innovation leads to positive skew, imitation leads again to negative skew.

What is the more likely interpretation of a positive skewness of the composite error term in stochastic production frontier analysis? On the one hand, it may be an unfortunate draw and the industry may be characterized by 'super efficiency' (or at least, the symmetric error term $v_{i}$ dominates the one-sided distributed $u_{i}$ ). On the other hand, the large majority of firms may be quite inefficient (like in Figure 3). The two interpretations are completely different, either indicating no efficiencies or large inefficiencies. An argument against the first interpretation is that relative productivities of plants are persistent over time (e.g. Bailey et al., 1992). In case there would have been no inefficiencies (i.e. the error term is 
determined completely by statistical noise $\varepsilon_{i}=v_{i}$ ) one would not expect such persistence, unless the statistical noise has strong autocorrelation.

\section{Conclusion}

An important methodological problem in stochastic frontier analysis has been the occurence of residuals being skewed in the 'wrong' direction. In case of production frontiers many times positively skewed residuals have been found, while in case of cost frontier negative skewnesses have been quite common. The traditional solution to the problem has been to argue that there are no inefficiencies and to put the variance of the one-sided distribution equal to zero. This solution fails to be convincing. This paper suggests a different solution: the one-sided distribution of inefficiencies may be negatively skewed (in case of production frontiers) or positively skewed (in case of cost frontiers). This does not imply that the traditional solution argueing for unfortunate sampling is impossible, but that a better approach to the stochastic frontier analysis, in which a comparison is made of several industries (or regions or time periods), is to use a distribution allowing for positive and negative skewness.

\section{References}

Aigner, D.J., C.A.K. Lovell and P. Schmidt, 1977, Formulation and estimation of stochastic frontier production function models, Journal of Econometrics 6, 21-37.

Bailey, M.N., C. Hulten and D. Campbell, 1992, Productivity dynamics in manufacturing plants, Brookings Paper on Economic Activity: Microeconomics 1992, 187-267.

Fan, Y., Q. Li and A. Weersink, 1996, Semiparametric estimation of stochastic production frontier models, Journal of Business and Economics Statistics 14, 460-468.

Green, A. and D. Mayes, 1991, Technical inefficiency in manufacturing industries, Economic Journal 101, 523-538.

Greene, W.H., 1990, A Gamma-distributed stochastic frontier model, Journal of Econometrics 46, 141164.

Hosking, J.R.M., 1990, L-moments: Analysis and estimation of distributions using linear combinations of order statistics, Journal of the Royal Statistical Society, Series B, 52, 105-124.

Johnson, N.L., S. Kotz and N. Balakrishnan, 1994, Continuous Univariate Distributions, Vol. 1, second edition, New York: John Wiley \& Sons.

Johnson, N.L., S. Kotz and A.W. Kemp, 1992, Univariate Discrete Distributions, second edition, New York: John Wiley \& Sons.

$\mathrm{Li}$, Q., 1996, Estimating a stochastic production frontier when the adjusted error is symmetric, Economics Letters 52, 221-228.

Mester, L.J., 1997, Measuring efficiency at U.S. banks: Accounting for heterogeneity is important, European Journal of Operational Research 98, 230-242.

Olson, J.A., P. Schmidt and D.M. Waldman, 1980, A Monte Carlo study of estimators of the stochastic frontier production function, Journal of Econometrics 13, 67-82.

Waldman, D.M., 1982, A stationary point for the stochastic frontier likelihood, Journal of Econometrics $18,275-279$ 